\title{
Improving Energy Efficiency of Virtual Resource Allocation in Cloud Datacenter
}

\author{
N. Mahendran' and T. Mekala ${ }^{2}$ \\ 'Department of Electronics and Communication Engineering, M. Kumarasamy College of Engineering, \\ Thalavapalayam - 639113, Karur, Tamil Nadu, India; mahe.sec@gmail.com \\ 2Department of Computer Science and Engineering, M. Kumarasamy College of Engineering, \\ Thalavapalayam - 639113, Karur, Tamil Nadu, India; mekalathangavel@gmail.com
}

\begin{abstract}
Objective: To minimize the energy frenzied around cloud datacenters. The proposed system provides a dynamic and continuous load prediction along with existing heuristic load prediction to improve the adaptation performance for green computing through Markov chaining of resources. Methodology: The trouble of energy competence through resource management for a large-scale cloud environment to facilitate hosts sites is resolute here. In apply there will be number of underutilized servers in the datacenter which guzzle bulky part of established energy. To minimize the energy frenzied around datacenters the existing system outlines a distributed middleware architecture and presenting one of its key elements, a gossip protocol, Skewness algorithm and load balancing that meets our design goals: justice of resource allocation with respect to hosted sites through overload escaping and efficient edition to load changes and scalability in terms of together the numeral of machines and sites thereby turning off the unused servers for green computing through Markov Chaining Model. This model is scalable enough to signify systems composed of thousands of resources and it makes potential to represent both physical and virtual wealth exploiting cloud explicit concepts such as the infrastructure elasticity. Results: To certify the model, simulation is conducted within the Network Simulator (NS) 2.28 have platform with GCC 4.3 and Fedora 13. The load prediction algorithm and Markov chain model achieves both overload avoidance and green computing for systems with multiresource limitations. Application: Green Wireless Network: To improve the energy efficient, data retrieval and resource service based on coaching, computing and networking in the area of Green Wireless. Green Big Data: To reduce the power consumption, big data merge the concept with green computing. Green job Scheduling: Each Server has different jobs, to save the energy server merged with green computing. Green Cloud data center: To use the green computing in cloud data center to increases the energy efficiency.
\end{abstract}

Keywords: Green Computing, Load Prediction, Markov Chaining, Virtualization

\section{Introduction}

To run a public cloud computing, a repair source will first need to classify the navy that will be vacant to enterprises that desire to place their workloads in the cloud. It works with check provider through numerous programs, most especially .Datacenter Services curriculum, to help guarantee a minimum level of cloud examine capabilities. Service providers must give cloud consumers transparency and visibility and include them in the tune definition for their public cloud. To finish this, service providers must be able to supply significant logs to cloud consumers. In general, services should have logs casing the subsequent areas:

- Identity and contact management,

- User actions monitoring,

- Change and arrangement management,

- Security and threat supervision, and

- Business connection and availability administration. 


\subsection{Cloud Computing}

Cloud is a computing model, not a technology. Virtually cloud computing is computing one or additional logical computers on peak of a physical computer. In this sculpt "patrons" stop into the "cloud" to admission industrial property which are priced and offered "on-demand". Basically, industrial possessions are borrowed and jointed among many renters greatly as apartments, agency space, or cargo space places are worn by tenants. Transported beyond an Internet relation, the "cloud" restores the company data server or midpoint distributing the equivalent force. Thus, Cloud is purely industrial forces sell \& conveyed above the Internet. Cloud sellers connects virtualization (one particular computer hosting numerous unique "virtual" servers), automatic provisioning (personage servers have software established robotically), and Internet connection knowledge's to give the examiner. These are not narrative methodizes but a fresh name sensible to an anthology of senior (though modernized) methodizes that are unwrapped, sell and conveyed in a novel way $\stackrel{1}{\text {. }}$

Cloud engages aggregation of numerous computing, cargo space and network possessions into a sole creature called "cloud" into which location-free computing is realized. Cloud is an accepted expansion of the virtualization, Utility Computing and Service-Oriented Architecture (SOA). Virtualization in computing is the manufacture of a fundamental description of OS, a storage device, a hardware platform or network capital. For instance, an exacting physical server or engine can be sliced into dissimilar virtual machines or VMs, each embodying dissimilar possessions like CPU cores, memory, disk, etc. Virtualization can be versioned as division of a largely in industrial project that includes autonomic compute, an enlargement in which the industrial environment will be competent to administer itself based on utility computing. The plan of virtualization is to consolidate managerial tasks while humanizing Cloud and virtualization are equal. Cloud is based upon motivating and assigning, storage and network services in a public multi-tenant atmosphere. Virtualization is a key enabler for cloud. At the same time, cloud is also an authoritative strength pulling virtualization into the venture.

\subsection{Classification of Clouds}

There are diverse Classification of clouds that you can promise to based on your satisfaction $\frac{2,3}{3}$.

\subsubsection{Public Cloud}

This type of model can be admission by each subscriber with an internet and admittance to the cloud computing space.

\subsubsection{Private Cloud}

This model is established for a particular organization or group and restrictions provided to just that group.

\subsubsection{Community Cloud}

This type is collective amongst two or more organizations that have analogous cloud needs.

\subsubsection{Hybrid Type}

The fundamentally a merging of at least two cloud types, where the clouds included are combination of private, community, or public ${ }^{4}$.

\section{Background}

\subsection{Types of Cloud Computing}

\subsubsection{Software as a Service-SaaS}

It gives all the procedure of an urbane customary request to numerous vendors and frequently thousands of clients, except during a Web browser, not a "locally-installed" application. Diminutive or refusal program is organization on the client's partial computer and the purposes are regularly modified to accomplish precise occupations. SaaS eradicates buyer qualms about submission storage, application development servers, and connected common fears of IT. Highest-profile patterns are Salesforce. com, Google's Gmail and Apps, Google and Yahoo, instant messaging from AOL, and Skype and VoIP from Vonage.

\subsubsection{Platform as a Service- PaaS}

Distributes virtualized servers on which patrons can sprint obtainable procedure or lengthen novel ones lacking having to agonize regarding keeps the server load balancing, operating systems, computing capacity or h/w. These sellers give APIs or enlarge platforms to generate and scuttle procedures in the cloud computing - e.g. with the Internet. Examine suppliers with appliance services suppliers to IT sections to supervise downstream applications and systems such as virus scanning for e-mail are 
habitually integrated in this group. Glowing branded suppliers would contain ADP Payroll processing, Salesforce. com, Google Maps, Microsoft's Azure, and US Postal Service offerings.

\subsubsection{Infracture as a Service-IaaS}

Distributes efficacy computing ability, naturally as unprocessed virtual servers, on demand service that customer organize and direct. Here Cloud offers clusters or grids or networks, storage virtualized servers and system software, regularly (but not forever) in a multitenant building. IaaS is considered to enhance or substitute the purposes of a complete data core. This keeps rate (outflow and time) of capital apparatus consumption but does not condense outlay of arrangement, incorporation or supervision and these errands must be performed tenuously.

\subsection{Trust in Cloud Computing}

While here is no collectively traditional designation of hope in cloud, it is significant to elucidate its apparatus and significance. In dictionaries, trust is usually correlated to "levels of assurance in amazing or a celebrity". Thus we can analysis hope in the cloud as the clients' height of confidence in with the cloud, and attempt to augment this by extenuating technical and emotional fences to with cloud forces. For additional psychiatry of the explanations of hope in cloud a mechanism of hope in Cloud to best moderate difficulties to confidence, we require to comprehend the main mechanism distressing cloud trust:

\subsubsection{Security}

Methods (e.g. encryption mechanism) are which arrange it enormously intricate or unschooled for an unconstitutional personality to admission various in sequence.

\subsubsection{Privacy}

Shield touching the experience or outflow of special or classified information (e.g. PII-personally identifiable information).

\subsubsection{Accountability}

Classified in as "the requirement and/ or compliance to display and take dependability for performance in light of agreed-upon opportunity", accountability goes outside dependability by forced a society to be responsible for its events. It has been traditional in direction by organiza- tion such as APEC, OECD, and PIPEDA as placing an officially authorizes liability upon an association that uses in person restricted in sequence to ensure that slender associates to whom it resources the PII are submissive to seclusion strategy, everywhere in the world they may be.

\subsubsection{Auditability}

The comparative relieve of checking a setting or a system. Pitiable auditability means that the system has threadbare (or unreal) account and systems that facilitate resourceful inspecting of progression within the cloud. It is also an enabler of (presentation) accountability: It permits an achievement to be analyzed alongside a prearranged strategy to choose if the achievement was submissive and if it was not, to grasp answerable the organization or person accountable for the achievement.

Designed this model that increasing condition of cloud computing erects up great number of great scale datacenters which needs great quantity of energy. This leads to depletions of usual authority more which is the rising complexity. They undertake power capability of virtual resource split for cloud as multi-objective optimization problem through bright optimization algorithm, Service Level Agreement (SLA) and Scheduling Virtual Machines (SVM) for load balancing to boost their routine. Energy optimization connection uses virtualization expertise; virtualization creates the operation of datacenters elastic, protected, fault tolerance, manageable and cheap. Further VM wares using virtualization technology combined OS since dissimilar OS environment be in same physical machine. The system utilizes Live Migration technology to travel within equal physical machine and from one physical machine to any more. They work on two classes: 1. Server Level Management to keep energy for one physical machine during Dynamic Voltage Frequency Scaling (DVFS), and 2. Cluster Level Management which presents overall energy economy by virtualization and server changing on/off during Bin Packing Algorithm ${ }^{5}$.

Proposed that in Utility based Computing business replica, the holders of the calculating resources discuss with their possible patrons to sell calculating control. The provisos of the QOS and the monetary condition are predictable in a Service-Level Agreement. Here are numerous situations in which the resolute QOS cannot be generous because of errors in the tune provisioning or stoppage in the method. Because suppliers have frequently unusual types of customers, according to their affiliation 
with the contributor or by the charge that they forfeit, it is noteworthy to diminish the collision of the SLA abuses in particular patrons. This addresses a tub of policies to grant healthier QoS to individual customers in such states. The theory to arrange customers is well-known according to the association among provider and client (external user, internal or another privileged connection) and the QoS that the consumer procures (shameful agreements or added QoS by paying an added cost). Mainly of the policies exercise key skin surface of virtualization: judicious abuse of the SLAs, vigorous Scaling of the payable Resources, and Runtime removal of Tasks ${ }^{6}$.

Deals that cloud is promising computing representations that can adapt the technique how in rank services are conditioned. Network virtualized acting a critical position in a cloud computing background for intangible and virtualizing diverse IAS. Therefore practical network forces obligation be incorporated with Cloud service to figure the merged Cloud service. Conversely, miniature research has aware on modeling and improvement of network virtualization with Cloud service provisioning to last part patrons. In this paper, representation the Cloud service conditioning quality in virtualization-based Cloud, and advice a proper algorithm for QoS awakes service work to better clients knowledge's for Cloud service admission. On the other hand, creates suitable service mechanism into a Cloud service that gathers numerous QoS provisions is a demanding optimization focus since such a trouble can be fundamentally addressed as Multi-Constraints Path (MCP) problem, which is recognized to be NP-hard. To handle this trouble, greatly discover growth has been made near dishonest capable algorithms recently. Xue defined a different report of MCP and available a group of irregular computation algorithms for every difficulty, which are identified as the finest amid the aforementioned outcomes. Huang told a heuristic for Multi-Constrained Optimal Path (MCOP) collection, in which a nonlinear mixture system and a geographical portrayal of a wellknown process in finding Delay Constrained Least Cost (DCLC) were started. Our imaginary scrutiny shows that the premeditated algorithm is cost-effective and lightweighted. The evaluation of planned algorithm beside a departure of the greatest branded QOS routing algorithm experimentally is finished. The consequences confirm that the projected algorithm has healthier prove both in completing time and verdict result. It's held that the coping procedure and the algorithm handy in this paper are widespread and valuable, thus are apposite to handy Cloud computing systems ${ }^{?}$.

\section{Existing System}

Resource organization is serious in cloud computing process. Among indecent applications strength practice network blocking, resource organization, CPU waste, stretched time wait, security troubles, boring CPU, and memory. To exploit cloud infrastructure consumption and decrease whole cost of together the running applications, and cloud infrastructure and possessions necessitate to be prohibited suitably. To conquer this there are types of possessions in the extensive calculating infrastructure necessitate to be controlled, disk quota, CPU load, network bandwidth, and level type of operating systems. To give superior QOS, resources are provisioned to the applications or users, via load balancing method, high availability method, and authority and security mechanism. To utilize cloud operation, the capability of application necessities shall be designed so that negligible cloud infrastructure approach will be procured and maintained. Known admission to the cloud infrastructure, submissions shall allocate accurate resources to achieve the addition with instance cost and transportation indict minimized.

Most of the cloud servers are underutilized; to reduce the electricity fee and hardware we provide the Virtual Machine Monitor (VMM) to atlas the parallel VM's to the physical resources. However this matching is being secreted beginning the cloud customers. It is awake to the cloud provider to construct positive that primary physical machine has adequate resource to assemble their wants. VM's survive movement technology modifies the mapping among PM's and VM's while the application is successively. SLA strategy decides the matching. The resource stress of VM is met while the use of physical machine is reduced. The resource wants of VM are mixed due to varied set of application they scamper since the applications differ with time and their workload produces and contract respectively. The capacity of PM's too mixed since various making hardware co-exists in datacenter $\stackrel{乛}{-}$.

The main goals addressed here are: 1. Green Computing, and 2. Overload avoidance. To poise those two objectives resource allowance system to shun overload in the system; idea of "skewness" to count unevenness; 
load forecast algorithm to forecast the outlook many of the VM.

Every PM runs a hypervisor VMM which chains essential one privileged field Dom $\mathrm{O}$ and one or more user defined field Dom U. The Dom U can be any one of the set of web server, DNS, mail, map/reduce, remote desktop, ect ... Beginning the architecture deduce that all the PM allocates the similar backend recall. Multiplexing every the VM's mutually to the PM is ready using guide Framework. Guide expresses the appeal to the great point. In PM each node runs a conduct Local Node Manager on Dom O; it saves all the custom figures of the possessions for every VM on that node. CPU and network custom are designed using arrangement measures in Xen. These are fundamentally designed since PM custom is probable with that. Memory custom are not evident to the hypervisor usually, with the help of exchange events we deduce memory shortage here. It gets too late to control recall share by time exchange. The WS-Prober on every hypervisor which are employed to estimation the operational position amount of VM organization on it. It exercises chance page variety method as in VMware ESX Server. Position of PM is advance to the guide controller. Guide controller is raised occasionally from LNM: 1. resource requires history of VM, 2. present explain of VM on PM, and 3. faculty and shipment account of VM on PM

Figure 1 shows that the scheduler is the secondary area of the Guide manager to execute the subsequent events.
Analyst predicts the resource on VM and the shipment on PM based on the history statistics. The generally pack on PM is the entirety aggregation of property on VM. LNM gratify the shipment weight nearby by correct the source of VM allocating the identical VMM. Xen be able to adjust the CPU portion between the VM by correcting heaviness in its CPU scheduler. MM assigner on Dom $\mathrm{O}$ is answerable for correcting limited memory portion. The hot mark resolver in our VM Scheduler spots if the reserve deployment of some PM is over the hot verge (i.e., a hot spot). If so, a few VMs organization on them will be transferred absent to scratch their load. The cold speck solver proves if the standard use of vigorously worn PMs (APMs) is beneath the green computing doorstep. If so, several of persons PMs might potentially be twisted off to save energy. It classifies the location of PMs whose operation is lower the cold entrance (i.e., cold sites) and then labors to journey gone all their VMs. It then assembles a relocation inventory of VMs and overtakes it to the conduct CTRL for completing.

\section{Problem Definition}

The proposed model judges the method of source supervision for a big-scale cloud background. Such a location comprises the physical transportation and related organize well-designed that permits the provisioning and supervision of cloud services. The discernment full is

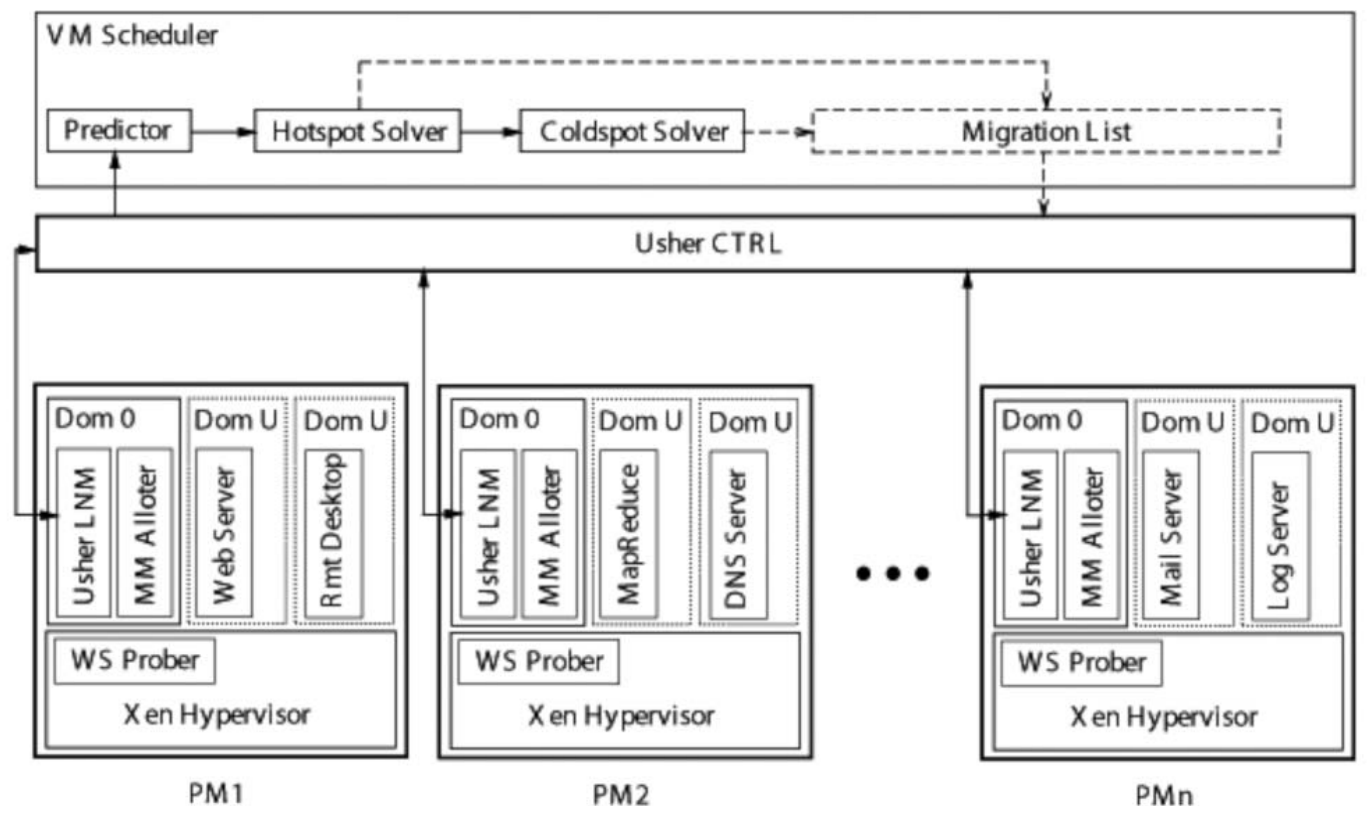

Figure 1. Virtual machine monitor. 
that of a cloud service spreader, which hosts spot in a cloud situation. The cloud service contributor holds and reins the physical carrying, on which cloud services are approved. It proposes hosting force to position holders during a middleware that effects on its transportation. Spot holders give services to their hold clients via spots that are crowded by the cloud tune contributor. Thus, the consumer anxieties are distorted to this practical cloud server. During this experienced scheme, the client's demands will be pleased fruitfully by serving the patron lacking waiting. So, the income will be owed animatedly. This job gives nears manufacturing a middleware level that executes resource portion in such a cloud situation, with the subsequent devise aims:

1. Performance: We believe memory possessions and computational and the point is to reach max-min equality for subtraction funds beneath memory checks,

2. Adaptability: The reserve allowance process should energetically and capably adjust to amends in the command for cloud forces, and

3. Scalability: The reserve portion progression necessity is scalable mutually in the numeral of apparatus in the cloud and the numeral of spots that the cloud. This funds that the possessions stimulated per machine in categorize to reach a given presentation purpose should boost sub linearly with both the numeral of equipment and the numeral of spots.

In this paper, the implementation and design of a computerized resource organization scheme that achieves a superior constancy between the two targets is presented. The subsequent contributions are:

1. Resource allowance system is urbanized that preserve evade overwork in the organism successfully while diminishing the numeral of servers worn,

2. The notion of "skewness" is established to quantify the rough operation of an attendant. By diminishing skewness, it progresses the generally utilize of servers in the expression of multiple- dimensional resource manacles, and

3. Calculated a load guess algorithm that can manage the expect reserve conventions of requests precisely with no seeming indoor the VMs. The algorithm can detain the growing tendency of source perform outlines and assist to decrease the assignment agitate appreciably.

\section{Proposed System}

A knowledge that is intelligent to advance the consumption of server wealth, and thus, diminish power spending, is virtualization of figuring possessions. Figure 2 shows that, virtualization commences an abstraction layer linking an OS and hardware. Physical property can be separating into a numeral of consistent pieces called VMs apiece VM container lodge a personality OS fashioning for the patron an examination of a fixated physical reserve and craft sure the management and stoppage disjointing among VMs allocation a fussy physical machine. The predicament of crowd overloads exposure as a measurement of dynamic DVM consolidation. Seminal when it is paramount to shunt VMs starting a crowded host is a feature of energetic VM consolidation that straight weights the reserve spending and Quality of Service transported by the association. The ability on the QOS is clarified by the information that server excesses reason reserve lacks and act squalor of applications. In close by responds to the complexity of host surplus discovery are characteristically heuristic-based, or rely on arithmetical psychiatry of precedent data. The restrictions of these advances are that they direct to suboptimal punishment and do not authorize unambiguous obligation of a QOS objective. A narrative advance that for several recognized motionless workload and an agreed state arrangement most favorably resolves the trouble of host surplus discovery by the exploiting denote inter-migration time below the particular QOS objective supported on a Markov chain model. The algorithm is heuristically modified to lever mysterious non-stationary workloads with the Multisite Sliding Window workload inference practice.

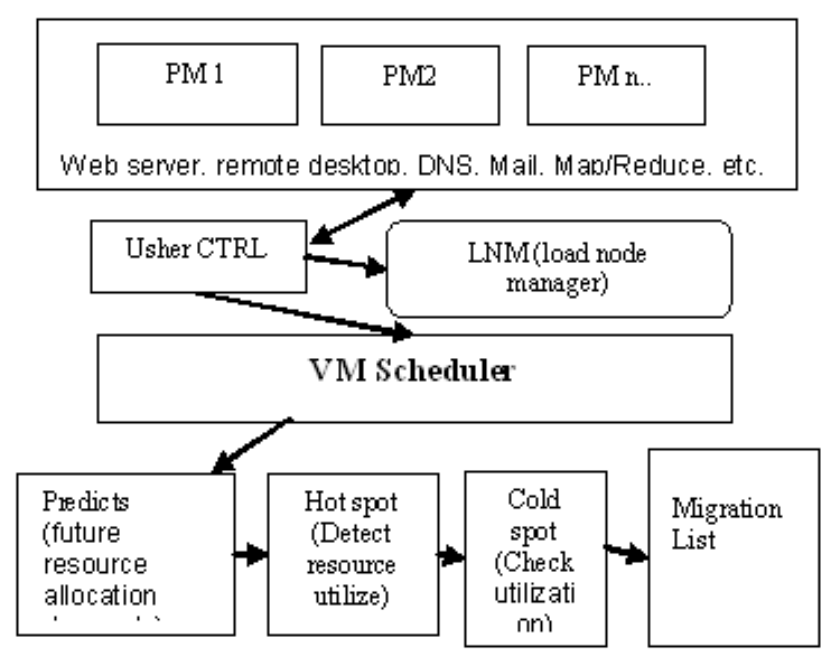

Figure 2. Virtual monitor. 
The key donations of this subdivision are the subsequent:

1. A systematic model performance that to recover the eminence of VM consolidation, it is obligatory to exploit the connote time connecting VM passages instigated by the host burden recognition algorithm,

2. A finest offline algorithm for parishioners overtaxes recognition, and substantiation of its finest,

3. A innovative Markov chain sculpt depiction that permits a opening of a randomized administer approach that optimally decides the problem of with the mean time amid VM exodus underneath an honestly tough QoS ambition for any notorious immobile work process and a notorious state pattern in the online scenery, and

4. A heuristically customized algorithm for behavior imprecise non-stationary workloads with the Multiple-size Sliding Window workload inference advance, which guides to equivalent to the finest benchmark algorithm routine in provisos of the intermigration time, while present the plus of particular pattern of a QOS ambition. Figure 3 shows that flow of working procedure of virtual machine.

\section{Performance Evaluation}

\subsection{Simulation Configuration}

To certify the model, simulation is performed inside the NS -Network Simulator 2.28 comprise platform among GCC 4.3 and Fedora 13. A laptop controls amid3-GB RAM and Core 2 Duo T7250 CPU. To receiving improved contrast simulation fallout with additional research mechanism, we approved to NS 2.28 evade situations. The meaning is to give more universal results and create it effortless for us to contrast the results. In NS 2.28, the default configuration identifies 60 nodes in a flat gap

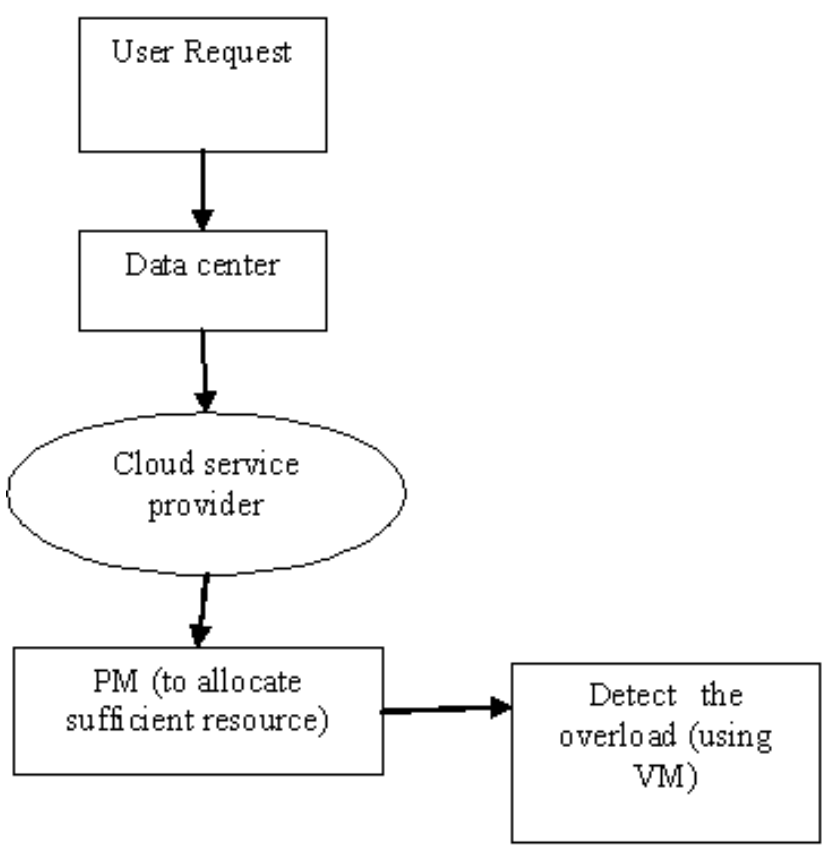

Figure 3. Machine monitor.

among a size of $670 \times 670 \mathrm{~m}$. The mainly hops are permissible in this configuration position are four.

\subsection{Effect of Thresholds on APMs}

We imitate a system with 1,000 VMs and $100 \mathrm{PMs}$ (selected arbitrarily from the outline). We utilize random PM to VM drawing in the untimely draft. The scheduler is bringing into play once per minute. The base part of Figure 4-5 shows the each day consignment divergence in the system. The $\mathrm{x}$-axis is the point of the day opening at 9 am. The $y$-axis is congested through 2 meanings: the $\%$ of APMs in the system or the \% of the load. Remember that a PM is vigorous (i.e., an APM) if it has at slightest one $\mathrm{VM}$ running. As can be seen starting the form, the CPU

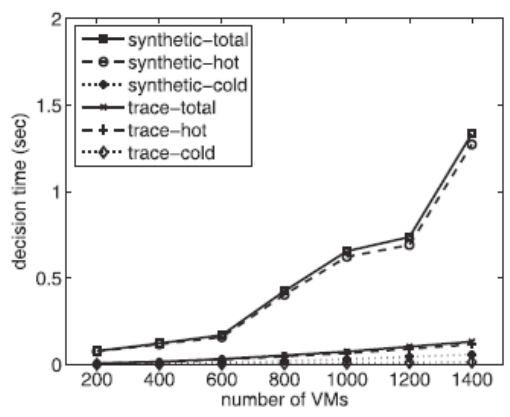

(a) average decision time

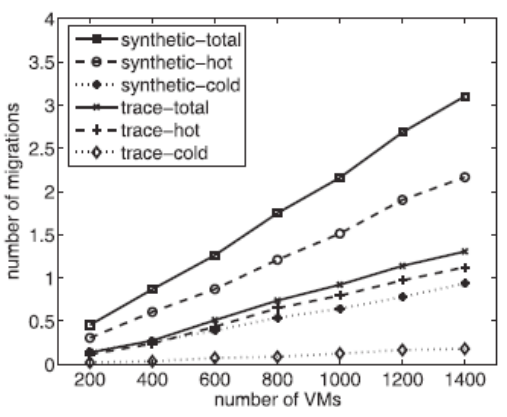

(b) average number of migrations

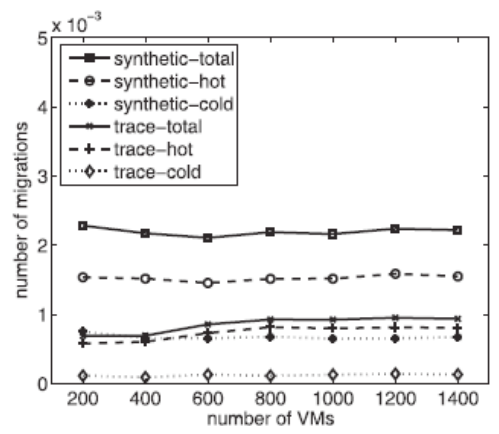

(c) number of migrations per VM

Figure 4. Existing result. 


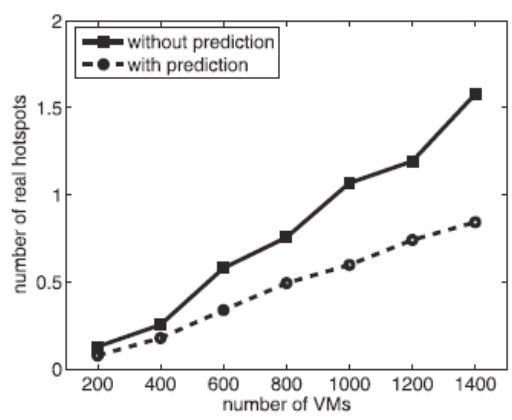

(a) number of hot spots

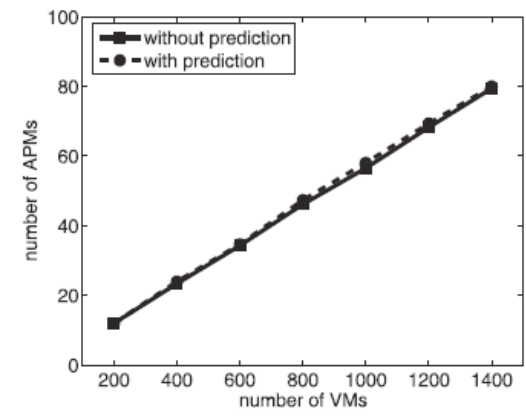

(b) number of APMs

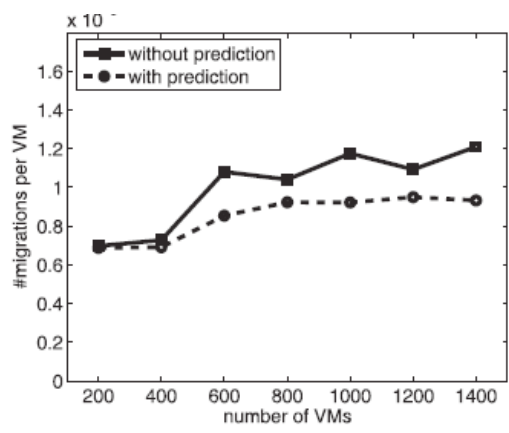

(c) number of migration

Figure 5. Proposed result.

freight elucidates a diurnal model which reduces considerably subsequent midnight. The memory expenditure is comparatively steady above the time. The network expenditure waits especially squat.

\section{Conclusion}

We include accessible the plan, accomplishment, and valuation of a reserve organization system for cloud services. Our system multiplexes implicit possessions to physical possessions adaptively standard on the altering control. We occupy the skewness metric to connect VMs among dissimilar reserve individuality properly so that the capability of server is glowing worn. It realizes together overwork averting and green computing for systems with multire source limitations.

\section{References}

1. Chen G, Wenbo H, Liu J, Nath S, Rigas L, Xiao L, Zhao F. Energy-Aware Server Provisioning and Load Dispatching for Connection-Intensive Internet Services. Proceedings USENIX Symposium Networked Systems Design and Implementation (NSDI '08); 2008. p. 337-50.

2. Mahendran N. Collaborative location based sleep scheduling with load balancing in sensor-cloud, International Journal of Computer Science and Information Security (IJCSIS). 2016; 14:20-27.
3. Mahendran N, Priya R. Sleep scheduling schemes based on location of mobile user in sensor-cloud, International Journal of Computer, Electrical, Automation, Control and Information Engineering. 2016; 10(3):615-20.

4. Armbrust M, Fox A, Griffith R, Joseph AD, Katz R, Konwinski A, Lee G, Patterson D, Rabkin A, Stoica I, Zaharia M. Above the Clouds: A Berkeley View of Cloud Computing. Technical Report No. UCB/EECS-2009-28; 2009. p. 1-25.

5. Xu L, Zeng Z, Ye X. Multi-Objective Optimization Based Virtual Resource Allocation Strategy for Cloud Computing. IEEE/ACIS International Conference on Computer and Information Science, China; 2012. p. 56-61.

6. Macias M, Guitart J. Client Classification Policies for SLA Enforcement in Shared Cloud Datacenters. IEEE/ACM Symposium on Cluster, Cloud and Grid Computing, Canada; 2012. p. 156-63. Crossref.

7. Huang J, Liu Y, Duan Q. Service provisioning in Virtualization-based Cloud Computing: Modeling and Optimization. Proceedings of Communications QoS, Reliability and Modeling Symposium; 2012. p.1728-33.

8. Barham P, Dragovic B, Fraser K, Hand S, Harris T, Ho A, Neugebauer R, Pratt I, Warfield A. Xen and the Art of Virtualization. Proceedings of ACM Symposium on Operating Systems Principles (SOSP '03), New York, USA; 2003. p. 1-14. Crossref. 